\title{
The analysis of the influence of structural parameters on static and dynamic properties of sliding bearings with a floating ring bearing
}

\author{
Aleksander Mazurkow \\ Rzeszow University of Technology, The Faculty of Mechanical Engineering and Aeronautics, \\ Dept. of Mechanical Engineering, Powstancow Warszawy Avenue 8, 35-959 Rzeszów, Poland \\ amazurkow@gmail.com
}

\begin{abstract}
Turbochargers structure developments i.e. work of rotating sets in turbochargers with higher rotational speed imposes more on bearing knots. The recommended values of parameters in this paper were introduced to describe bearings with floating rings. The authors used computational examples to show the influence of presented ranges of a relative clearance of the inner bearing and quotients of radial clearances on work parameters of a sliding bearing with a floating ring such as: relative eccentricities, oil films maximum temperatures, oil films maximum pressures, oil films minimal heights, a journal velocity quotient and a floating ring as well as amplitudes of displacements of floating rings in bearing knots of a rotating set in a turbocharger.
\end{abstract}

Keywords: Turbocharger, rotating unit, oil film force, adiabatic model, eccentricity ratio.

\section{Introduction}

Turbochargers structure developments i.e. work of rotating sets in turbochargers with higher rotational speed imposes higher requirements on bearing knots (Fig.1). A bearing shaft described in Fig. 1 is bearing mounted in lateral sliding bearings with a floating ring (Fig. 3). The right choice of structural characteristics of sliding bearings in an essential way influences the proper work of rotating sets. Therefore, we must use the appropriate design methods. Theoretical and experimental models constitute the base for these methods. These models should reflect very precisely the real conditions of work in a bearing.

Taking into account the results from the study of static and dynamic properties in the research Mazurkow (2009a) authors have recommended the following values of parameters which describe the geometry of sliding bearings with floating rings:

$\psi_{1}=\frac{C_{R 1}}{R_{1}}=\frac{R_{1}-R_{J 1}}{R_{1}} \approx 4-5 \%$, relative clearance of

inner oil film,

$$
C_{R}^{*}=\frac{C_{R 2}}{C_{R 1}}=\frac{R_{2}-R_{J 2}}{R_{1}-R_{J 1}}=0,5-1,0, \text { quotient of radial }
$$

ranges of a relative clearance of the inner bearing $\left(\psi_{1}\right.$ and quotients of radial clearances $\left(\mathrm{C}_{\mathrm{R}}^{*}\right)$ on work parameters of a sliding bearing with a floating ring such as: relative eccentricities $\left(\varepsilon_{1} \& \varepsilon_{2}\right)$, maximum temperatures of oil films

$\left(T_{1 \max } \& T_{2 \max }\right)$, maximum pressures of oil films ( $p_{1 \max }$, $\left.\mathrm{p}_{2 \max }\right)$, minimal heights of oil films $\left(\mathrm{h}_{1 \min } \& \mathrm{~h}_{2 \min }\right)$, a velocity quotient of a journal and a floating ring $\left(v=\omega_{1} / \omega_{2}\right)$ as well as amplitudes of displacements of floating rings in bearing knots of a rotating set in a turbocharger: $\left(\mathrm{x}_{5}, \mathrm{y}_{5}, \mathrm{x}_{6} \& \mathrm{y}_{6}\right)$.

Dynamic model of a rotating set in a turbocharger

To deal with the subject the authors have developed the dynamic model of a rotating set in a turbocharger which is described in the Fig. 2. It has been accepted that rotors on either a turbine or a compressor side have different masses and different unbalance values. In this model each bearing is modeled including a floating ring bearing mass. Through rigidity and damping factors oil films capacity towards oscillations damping is also considered. The motion of the model is examined in two planes: $O X Z$ and $O Y Z$. Here, presented rigidity factors $\left(C_{x}\right.$ $\left.\& c_{y}\right)$ and damping factors $\left(d_{x} \& d_{y}\right)$ are studied as coupled values.

A mathematical model of a presented physics model is constituted by motion equations which are written according to forces method for each mass of this model in planes $O X Z$ and $O Y Z$ and can be expressed: clearance, (1)

$$
B^{*}=\frac{B}{D_{1}}=\frac{B}{2 \cdot R_{1}} \leq 0,5,
$$

bearing bush relative width.

The aim of this research work is to use calculation examples and discuss the influence of presented

$$
\begin{aligned}
& x_{1}=-\alpha_{x 11}\left[m_{1} \ddot{x}_{1}-m_{n 1} \delta_{1} \omega^{2} \sin \omega t\right]-\alpha_{x 12}\left[m_{2} \ddot{x}_{2}+d_{x 2}\left(\dot{x}_{2}-\dot{x}_{5}\right)\right]-\alpha_{x 13}\left[m_{3} \ddot{x}_{3}+d_{x 3}\left(\dot{x}_{3}-\dot{x}_{6}\right)\right]- \\
& -\alpha_{x 14}\left[m_{4} \ddot{x}_{4}-m_{n 4} \delta_{4} \omega^{2} \sin \omega t\right] \\
& x_{2}=-\alpha_{x 21}\left[m_{1} \ddot{x}_{1}-m_{n 1} \delta_{1} \omega^{2} \sin \omega t\right]-\alpha_{x 22}\left[m_{2} \ddot{x}_{2}+d_{x 2}\left(\dot{x}_{2}-\dot{x}_{5}\right)\right]-\alpha_{x 23}\left[m_{3} \ddot{x}_{3}+d_{x 3}\left(\dot{x}_{3}-\dot{x}_{6}\right)\right]- \\
& -\alpha_{x 24}\left[m_{4} \ddot{x}_{4}-m_{n 4} \delta_{4} \omega^{2} \sin \omega t\right] \\
& x_{3}=-\alpha_{x 31}\left[m_{1} \ddot{x}_{1}-m_{n 1} \delta_{1} \omega^{2} \sin \omega t\right]-\alpha_{32}\left[m_{2} \ddot{x}_{2}+d_{x 2}\left(\dot{x}_{2}-\dot{x}_{5}\right)\right]-\alpha_{x 33}\left[m_{3} \ddot{x}_{3}+d_{x 3}\left(\dot{x}_{3}-\dot{x}_{6}\right)\right]- \\
& -\alpha_{x 34}\left[m_{4} \ddot{x}_{4}-m_{n 4} \delta_{4} \omega^{2} \sin \omega t\right]
\end{aligned}
$$

"Turbocharger: design improvement" http://www.indjst.org
A. Mazurkow Indian J.Sci.Technol. 


$$
\begin{aligned}
& x_{4}=-\alpha_{x 41}\left[m_{1} \ddot{x}_{1}-m_{n 1} \delta_{1} \omega^{2} \sin \omega t\right]-\alpha_{x 42}\left[m_{2} \ddot{x}_{2}+d_{x 2}\left(\dot{x}_{2}-\dot{x}_{5}\right)\right]-\alpha_{x 43}\left[m_{3} \ddot{x}_{3}+d_{x 3}\left(\dot{x}_{3}-\dot{x}_{6}\right)\right]- \\
& -\alpha_{x 44}\left[m_{4} \ddot{x}_{4}-m_{n 4} \delta_{4} \omega^{2} \sin \omega t\right] \\
& m_{5} \ddot{x}_{5}+c_{x 4} x_{5}+d_{x 4} \dot{x}_{5}+c_{x 2}\left(x_{5}-x_{2}\right)+d_{x 2}\left(\dot{x}_{5}-\dot{x}_{2}\right)=0 \\
& m_{6} \ddot{x}_{6}+c_{x 6} x_{6}+d_{x 6} \dot{x}_{6}+c_{x 3}\left(x_{6}-x_{3}\right)+d_{x 3}\left(\dot{x}_{6}-\dot{x}_{3}\right)=0 \\
& y_{1}=-\alpha_{x 11}\left[m_{1} \ddot{y}_{1}-m_{n 1} \delta_{1} \omega^{2} \sin \omega t+Q_{1}\right]-\alpha_{x 12}\left[m_{2} \ddot{y}_{2}+d_{x 2}\left(\dot{y}_{2}-\dot{y}_{5}\right)+Q_{2}\right]- \\
& -\alpha_{x 13}\left[m_{3} \ddot{y}_{3}+d_{x 3}\left(\ddot{y}_{3}-\ddot{y}_{6}\right)+Q_{3}\right]-\alpha_{x 14}\left[m_{4} \ddot{y}_{4}-m_{n 4} \delta_{4} \omega^{2} \sin \omega t+Q_{4}\right] \\
& y_{2}=-\alpha_{x 21}\left[m_{1} \ddot{y}_{1}-m_{n 1} \delta_{1} \omega^{2} \sin \omega t+Q_{1}\right]-\alpha_{x 22}\left[m_{2} \ddot{y}_{2}+d_{x 2}\left(\dot{y}_{2}-\dot{y}_{5}\right)+Q_{2}\right]- \\
& -\alpha_{x 23}\left[m_{3} \ddot{y}_{3}+d_{x 3}\left(\ddot{y}_{3}-\ddot{y}_{6}\right)+Q_{3}\right]-\alpha_{x 24}\left[m_{4} \ddot{y}_{4}-m_{n 4} \delta_{4} \omega^{2} \sin \omega t+Q_{4}\right] \\
& y_{3}=-\alpha_{x 31}\left[m_{1} \ddot{y}_{1}-m_{n 1} \delta_{1} \omega^{2} \sin \omega t+Q_{1}\right]-\alpha_{x 32}\left[m_{2} \ddot{y}_{2}+d_{x 2}\left(\dot{y}_{2}-\dot{y}_{5}\right)+Q_{2}\right]- \\
& -\alpha_{x 33}\left[m_{3} \ddot{y}_{3}+d_{x 3}\left(\ddot{y}_{3}-\ddot{y}_{6}\right)+Q_{3}\right]-\alpha_{x 34}\left[m_{4} \ddot{y}_{4}-m_{n 4} \delta \omega^{2} \sin \omega t+Q_{4}\right] \\
& y_{4}=-\alpha_{x 41}\left[m_{1} \ddot{y}_{1}-m_{n 1} \delta_{1} \omega^{2} \sin \omega t+Q_{1}\right]-\alpha_{x 42}\left[m_{2} \ddot{y}_{2}+d_{x 2}\left(\dot{y}_{2}-\dot{y}_{5}\right)+Q_{2}\right]- \\
& -\alpha_{x 43}\left[m_{3} \ddot{y}_{3}+d_{x 3}\left(\ddot{y}_{3}-\ddot{y}_{6}\right)+Q_{3}\right]-\alpha_{x 44}\left[m_{4} \ddot{y}_{4}-m_{n 4} \delta_{4} \omega^{2} \sin \omega t+Q_{4}\right] \\
& m_{5} \ddot{y}_{5}+c_{y 4} y_{5}+d_{y 4} \dot{y}_{5}+c_{y 2}\left(y_{5}-y_{2}\right)+d_{y 2}\left(\dot{y}_{5}-\dot{y}_{2}\right)=Q_{5} \\
& m_{6} \ddot{y}_{6}+c_{y 6} y_{6}+d_{y 6} \dot{y}_{6}+c_{y 3}\left(y_{6}-y_{3}\right)+d_{y 3}\left(\dot{y}_{6}-\dot{y}_{3}\right)=Q_{6}
\end{aligned}
$$

The detailed discussion concerning the algorithm and the method of solving eqns. (2) is presented in works (Mazurkow, 2008; Mazurkow, 2009a).

Adiabatic model of a sliding bearing with a floating ring bearing

Oil to both bearings on a compressor and a turbine sides is supplied through a feeding duct which is in the middle frame. The structure of tubules which provide fresh oil causes that bearings are fed independently with a constant temperature and a constant feeding pressure $\left(T_{z} \& p_{z}\right)$. The detailed description of a structure and parameters which describe work of sliding bearings with floating rings (Fig. 3) is discussed in researches (Buluschek, 1980; Mazurkow, 1993; Mazurkow, 2008).

The adiabatic model of a short bearing has been accepted for calculations in this paper. This model is described in a dimensionless way by the following equations:

Equations of pressures distribution in lubricant gaps ( $i=1$, 2):

$\frac{\partial^{2} p_{i}^{*}}{\partial z^{* 2}}=6 \cdot \frac{\eta^{*}}{h_{i}^{* 3}} \cdot \frac{\partial h_{i}^{*}}{\partial \varphi_{i}} \cdot\left(\frac{B}{D_{i}}\right)^{2}$,

where: $p_{i}^{*}=p_{i} \cdot \frac{\psi_{i}^{2}}{\eta_{0} \cdot\left(\omega_{J i}+\omega_{i}\right)}$,

Equations of lubricant gaps shapes (i= 1, 2):

$\mathrm{h}_{i}^{*}=\frac{h_{i}}{C_{R i}}=1-\varepsilon_{i} \cdot \cos \left(\beta_{\mathrm{i}}-\varphi_{\mathrm{i}}\right)$,

Equations of temperature distribution in lubricant gaps (i= 1, 2):

Research article

(C)Indian Society for Education and Environment (iSee)
$C_{z i}\left(\varphi_{i}, z^{*} ; \eta^{*}\right) \cdot \frac{\partial T_{i}^{*}}{\partial z^{*}}=A_{z i}\left(\varphi_{i}, z^{*} ; \eta^{*}\right)$,

Where:

$$
\begin{aligned}
& A_{z 1}=-\left(\frac{\partial p_{1}^{*}}{\partial \varphi_{1}}\right)^{2}-\frac{12 \cdot \eta^{* 2}}{h_{1}^{*}} \cdot \frac{\left(\omega_{1} \cdot R_{1}-\omega_{2} \cdot R_{2}\right)^{2}}{R_{2}^{2}\left(\omega_{1}+\omega_{2}\right)^{2}}-\left(\frac{2 \cdot R_{2}}{B}\right)^{2} \cdot\left(\frac{\partial p_{1}^{*}}{\partial z^{*}}\right)^{2} \\
& A_{z 2}=-\left(\frac{\partial p_{2}^{*}}{\partial \varphi_{2}}\right)^{2}-\frac{12 \cdot \eta^{* 2}}{h_{2}^{* 4}}-\left(\frac{2 \cdot R_{4}}{B}\right)^{2} \cdot\left(\frac{\partial p_{2}^{*}}{\partial z^{*}}\right)^{2}, \\
& C_{z i}=\left(\frac{2 \cdot R_{i}}{B}\right)^{2} \cdot \frac{\partial p_{i}^{*}}{\partial z}{ }^{*}
\end{aligned}
$$

Equations of balance of powers and frictional moments:

$$
F_{L 1 y}^{*}=F_{1}^{*}, F_{L 2 y}^{*}=F_{2}^{*}, F_{L 1 x}^{*}=0, F_{L 2 x}^{*}=0
$$

Where:

$$
F_{1}^{*}=\frac{2 \cdot \psi_{1}^{2}}{\mathrm{~B} \cdot \mathrm{R}_{1} \cdot \eta_{0} \cdot\left(\omega_{1}+\omega_{2}\right)} \cdot \mathrm{F}, \quad F_{2}^{*}=\frac{2 \cdot \psi_{2}^{2}}{\mathrm{~B} \cdot \mathrm{R}_{3} \cdot \eta_{0} \cdot \omega_{2}} \cdot \mathrm{F}
$$

Equation of frictional moments balance in a bearing,

$$
\begin{aligned}
& M_{2}^{*}=M_{3}^{*}, \\
& \alpha_{2} \int_{-1}^{1} \int_{0}^{2 \pi}\left(\frac{1}{2} \cdot h_{2}^{*} \cdot \frac{\partial p_{2}^{*}}{\partial \varphi_{2}}+\frac{\eta^{*}}{h_{2}^{*}}\right) d \varphi_{2} d z^{*}= \\
& =\alpha_{1} \cdot \frac{\mathrm{R}_{1}}{\mathrm{R}_{3}} \int_{-1}^{1} \int_{0}^{2 \pi}\left(\frac{1}{2} \cdot h_{1}^{*} \cdot \frac{\partial p_{1}^{*}}{\partial \varphi_{1}}+\frac{\eta^{*}}{h_{1}^{*}} \cdot \alpha_{3}\right) d \varphi_{1} d z^{*}+\psi_{1} \cdot \varepsilon_{1} \cdot \sin \beta_{1}
\end{aligned}
$$


Fig. 1. A turbocharger with rotors: the centrifugal rotor of a compressor \& the centripetal rotor of a turbine
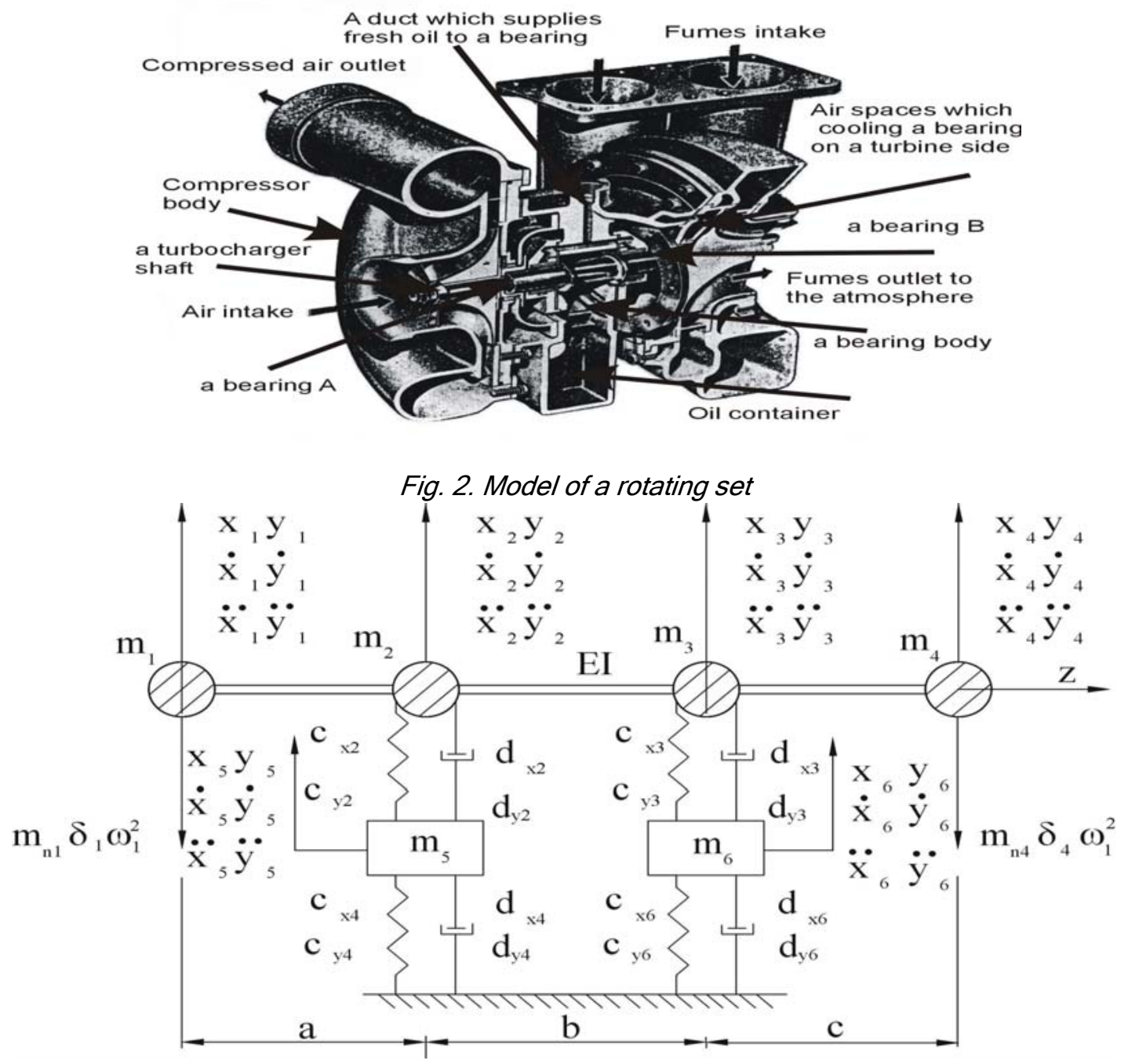

Fig. 3. A sliding bearing with a floating ring: 1-a floating ring bearing, 2-a fixed bearing bush, 3-holes through which oil is supplied to a bearing, 4-a circumferential groove, 5-directions of oil flow in a bearing

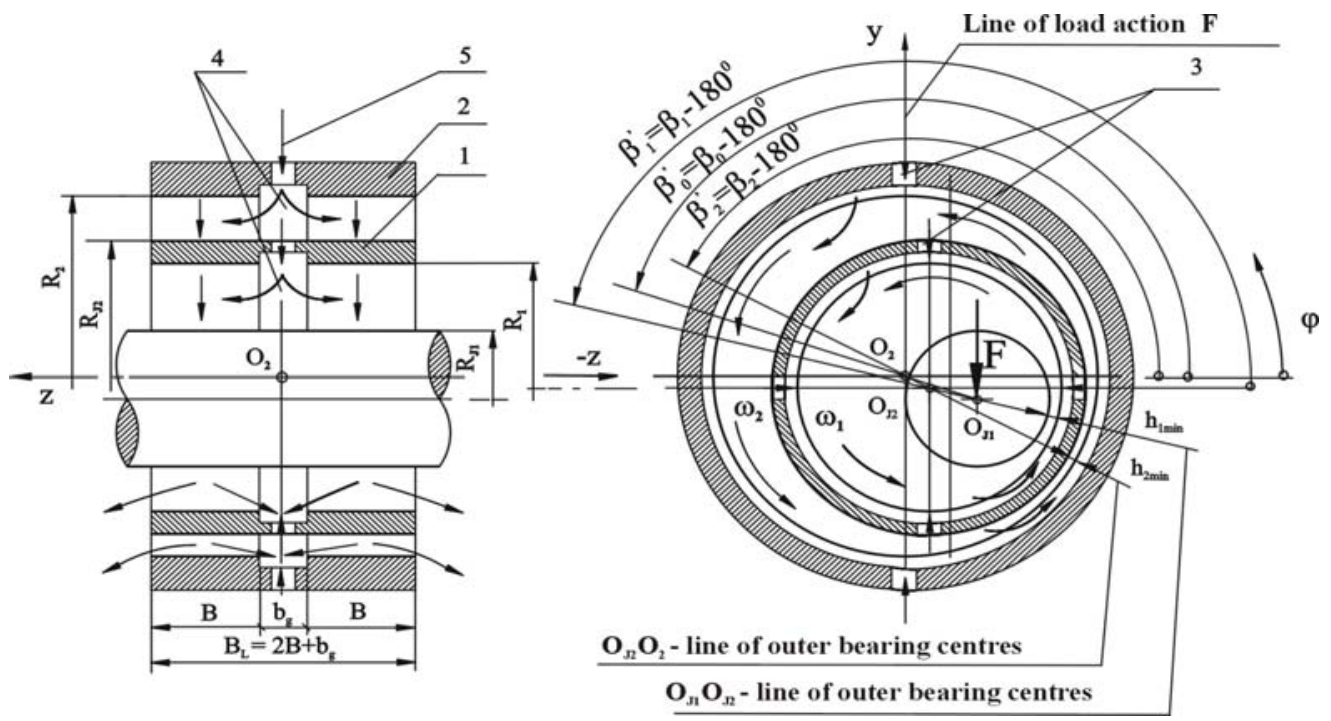

Research article

CIndian Society for Education and Environment (iSee)
"Turbocharger: design improvement" http://www.indjst.org
A. Mazurkow Indian J.Sci.Technol. 
Table 1. Given parameters in a short bearing model

\begin{tabular}{|c|c|}
\hline $\begin{array}{l}\text { Geometric } \\
\text { parameters }\end{array}$ & $\begin{array}{l}\text { Parameters concerning } \\
\text { bearing work }\end{array}$ \\
\hline $\begin{array}{c}\mathrm{R}_{\mathrm{Ji}}-\text { radius of bearing } \\
\text { journals, }\end{array}$ & F- bearing load, \\
\hline \multirow{5}{*}{$\begin{array}{c}R_{i} \text { - inner radius of } \\
\text { bearing bushes }\end{array}$} & $\begin{array}{l}\omega_{1} \text { - angular speed of bearing } \\
\text { journal }\end{array}$ \\
\hline & $T_{0}$ - reference temperature \\
\hline & $T_{z^{-}}$feed oil temperature \\
\hline & $\eta=\eta(T)$ - oil dynamic viscosity \\
\hline & $\begin{array}{l}c_{p}, \rho, \lambda \text {-specific heat, density, } \\
\text { oil conductivity. }\end{array}$ \\
\hline
\end{tabular}

Table 2. Influence of structural parameters on static \& dynamic characteristics

\begin{tabular}{|l|l|l|l|}
\hline \multicolumn{4}{|c|}{ Characteristics } \\
\hline \multicolumn{3}{|c|}{ Static } & \multicolumn{1}{c|}{ Dynamic } \\
\hline \multicolumn{1}{|c|}{$\mathrm{h}_{1,2 \text { min }} \downarrow$} & $\mathrm{T}_{1,2 \max } \downarrow$ & $\mathrm{p}_{1,2 \max } \downarrow$ & $\mathrm{A}_{\mathrm{p}}\left(\mathrm{x}_{5}, \mathrm{x}_{6}, \mathrm{y}_{5}, \mathrm{y}_{6}\right) \downarrow$ \\
\hline$\psi_{i} \downarrow$ & $\psi_{1} \downarrow$ & $\psi_{\mathrm{i}} \uparrow$ & $\psi_{\mathrm{i}} \downarrow$ \\
\hline $\mathrm{C}_{\mathrm{R}}^{*} \downarrow$ & $\mathrm{C}_{\mathrm{R}}^{*} \uparrow$ & $\mathrm{C}_{\mathrm{R}}^{*} \uparrow$ & $\mathrm{C}_{\mathrm{R}}^{*} \downarrow$ \\
\hline & & & $\mathrm{m}_{\mathrm{p}}=\mathrm{m}_{5}=\mathrm{m}_{6} \uparrow$ \\
\hline $\mathrm{B}^{*} \downarrow$ & $\mathrm{B}^{*} \uparrow$ & $\mathrm{B}^{*} \uparrow$ & $\mathrm{B}^{*} \uparrow$ \\
\hline & & & $\mathrm{N}_{\mathrm{w} \downarrow} \downarrow, \mathrm{N}_{\mathrm{w} 4} \downarrow$ \\
\hline $\mathrm{T}_{\mathrm{z}} \uparrow$ & $\mathrm{T}_{\mathrm{z}} \downarrow$ & $\mathrm{T}_{\mathrm{z}} \downarrow$ & \\
\hline
\end{tabular}

Table 3. Given parameters for calculations of sliding bearings with floating rings

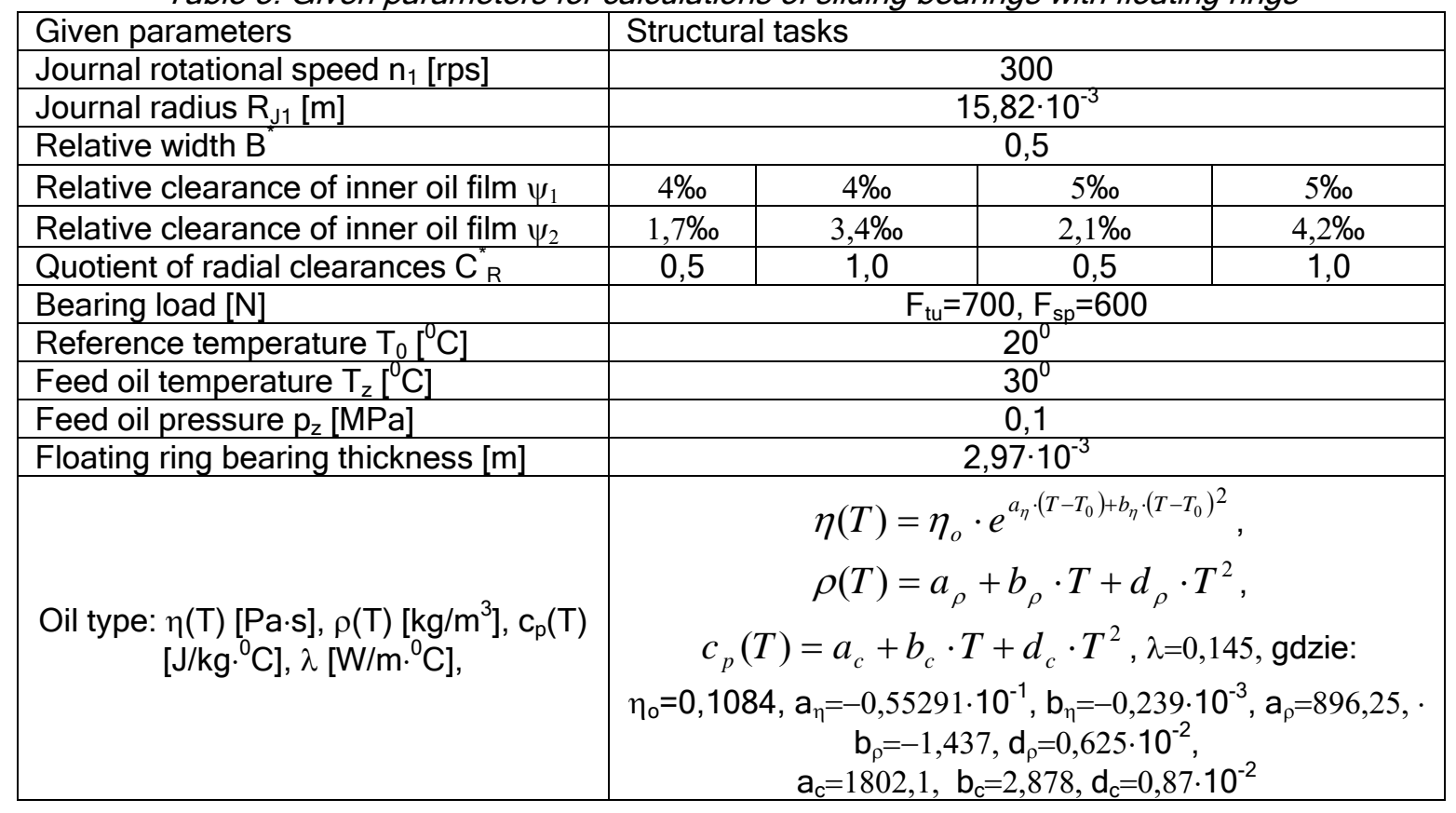

Table 4. Given parameters for calculations of the rotating set

\begin{tabular}{|c|c|c|c|c|c|}
\hline \multicolumn{6}{|c|}{ Concentrated masses in particular knots [kg] } \\
\hline $\mathrm{m}_{1}$ & $\mathrm{~m}_{2}$ & $\mathrm{~m}_{3}$ & $\mathrm{~m}_{4}$ & $\mathrm{~m}_{5}$ & $\mathrm{~m}_{6}$ \\
\hline 5,0 & 0,3 & 0,25 & 2,0 & 0,055 & 0,054 \\
\hline \multicolumn{6}{|c|}{ Geometric parameters of a rotating set } \\
\hline $\begin{array}{c}\mathrm{a}=0,055 \\
{[\mathrm{~m}]}\end{array}$ & $\begin{array}{c}\mathrm{b}=0,075 \\
{[\mathrm{~m}]}\end{array}$ & $\mathrm{c}=0,045[\mathrm{~m}]$ & $\mathrm{I}_{\mathrm{x}}=0,15 \cdot 10^{-6}\left[\mathrm{~m}^{4}\right]$ \\
\hline \multicolumn{6}{|c|}{ Young Modulus } \\
\hline \multicolumn{6}{|c|}{$\mathrm{E}=1,915 \cdot 10^{11}\left[\mathrm{~N} / \mathrm{m}^{2}\right]$} \\
\hline \multicolumn{6}{|c|}{$\mathrm{N}_{\mathrm{w} 1}=0,18 \cdot 10^{-4}$} \\
\hline
\end{tabular}

Research article

CIndian Society for Education and Environment (iSee)
"Turbocharger: design improvement" http://www.indjst.org
A. Mazurkow Indian J.Sci.Technol. 
Indian Journal of Science and Technology

Where calculations respectively amount to:

$\alpha_{1}=\frac{R_{1} \cdot \eta_{0} \cdot\left(\omega_{1}+\omega_{2}\right)}{\psi_{1}} \cdot \frac{B}{F}, \alpha_{2}=\frac{R_{3} \cdot \eta_{0} \cdot \omega_{2}}{\psi_{2}} \cdot \frac{B}{F}, \alpha_{3}=\frac{R_{1} \cdot \omega_{1}-R_{2} \cdot \omega_{2}}{R_{1} \cdot\left(\omega_{1}+\omega_{2}\right)}$

Vol. 3 No. 6 (June 2010)

ISSN: 0974- 6846

The following resultant parameters are searched:

Distributions of pressure and temperature:

$$
p_{i}^{*}=p_{i}^{*}\left(\varphi_{i}, z^{*}\right), T_{i}^{*}=T_{i}^{*}\left(\varphi_{i}, z^{*}\right)
$$

For non- working zone: $\varphi_{i}^{p}<\varphi_{i}<\varphi_{i}^{k}, \frac{\partial p_{i}^{*}}{\partial \varphi_{i}}=0$

For working zone:

- Structural characteristics such as:

- Angular speed of a floating ring $\left(\omega_{2}\right)$.

- Motion resistances in a bearing $\left(\mu_{\mathrm{i}}\right)$.

- Oil consumption which flows through a bearing $\left(\mathrm{Q}_{\mathrm{boi}}\right)$.

$\varphi_{i}^{k} \leq \varphi_{i} \leq \varphi_{i}^{p}, \frac{\partial p_{i}^{*}}{\partial \varphi_{i}}=A_{i} \cdot\left(z^{* 2}-1\right) \cdot\left[\frac{\cos \varphi_{i} \cdot\left(1-\varepsilon_{i} \cdot \cos \varphi_{i}\right)^{3}-3\left(1-\varepsilon_{i} \cdot \cos \varphi_{i}\right)^{2} \cdot \varepsilon_{i} \cdot \sin \varphi_{i}}{\left(1-\varepsilon_{i} \cdot \cos \varphi_{i}\right)^{6}}\right]$

- Positions of centres' lines: a journal, a floating ring bearing, a fixed bearing bush $\left(\varepsilon_{1}, \varepsilon_{2}, \varepsilon_{0}, \beta_{1}, \beta_{2}, \beta_{0}\right)$.

- Speed quotient of a bearing

The described above mathematical model of a bearing constitutes the coupled system of equations. This system of equations has been solved in a numerical way, the algorithm and the method of solving equations has been discussed in work (Mazurkow, 2009a).

journal and a floating ring $v=\omega_{1} / \omega_{2}$

Other models of a sliding bearing with a floating ring namely: the adiabatic model of a finite-length bearing, the isothermal model of a finite-length bearing and the isothermal one of a short bearing are described in these works (Buluschek, 1980; Domes, 1980; Krause, 1987;

Table 1 depicts accepted parameters. Mazurkow, 1993; Mazurkow, 2009a).

Table 5. Resultant parameters

\begin{tabular}{|c|c|c|c|c|}
\hline \multicolumn{5}{|l|}{ Resultant parameters, turbine side $\mathrm{F}_{\mathrm{tu}}=700[\mathrm{~N}]$} \\
\hline Structural tasks & $\begin{array}{l}1: \psi_{1}=4 \% \\
C_{R}^{*}=0,5\end{array}$ & $\begin{array}{l}2: \psi_{1}=4 \% \\
C_{R}^{*}=1,0\end{array}$ & $\begin{array}{l}3: \psi_{1}=5 \% \\
C_{R}^{*}=0,5\end{array}$ & $\begin{array}{l}4: \psi_{1}=5 \% \\
\mathrm{C}_{\mathrm{R}}^{*}=1,0\end{array}$ \\
\hline $\begin{array}{l}\text { Amplitudes of displacements of a floating ring } \\
10^{-6}[\mathrm{~m}]\end{array}$ & $\begin{array}{l}x_{5}=0,140 \\
y_{5}=0,250\end{array}$ & $\begin{array}{l}x_{5}=0,380 \\
y_{5}=1,210\end{array}$ & $\begin{array}{l}x_{5}=0,161 \\
y_{5}=0,409\end{array}$ & $\begin{array}{l}x_{5}=0,350 \\
y_{5}=1,510\end{array}$ \\
\hline Relative eccentricities & $\begin{array}{l}\varepsilon_{1}=0,29 \\
\varepsilon_{2}=0,25\end{array}$ & $\begin{array}{l}\varepsilon_{1}=0,25 \\
\varepsilon_{2}=0,48\end{array}$ & $\begin{array}{l}\varepsilon_{1}=0,42 \\
\varepsilon_{2}=0,416\end{array}$ & \begin{tabular}{|l|}
$\varepsilon_{1}=0,41$ \\
$\varepsilon_{2}=0,54$ \\
\end{tabular} \\
\hline Oil films maximum temperatures $\left[{ }^{0} \mathrm{C}\right]$ & $\begin{array}{l}T_{1 \max }=58 \\
T_{2 \max }=64\end{array}$ & $\begin{array}{l}T_{1 \max }=55 \\
T_{2 \max }=55\end{array}$ & $\begin{array}{l}T_{1 \max }=53 \\
T_{2 \max }=59\end{array}$ & $\begin{array}{l}T_{1 \max }=48 \\
T_{2 \max }=55\end{array}$ \\
\hline Oil films maximum pressures [MPa] & $\begin{array}{l}p_{1 \max }=4,0 \\
p_{2 \max }=2,5\end{array}$ & $\begin{array}{l}p_{1 \max }=3,2 \\
p_{2 \max }=2,6\end{array}$ & $\begin{array}{l}p_{1 \max }=4,5 \\
p_{2 \max }=3,4\end{array}$ & $\begin{array}{l}p_{1 \max }=5,4 \\
p_{2 \max }=3,6\end{array}$ \\
\hline Oil films minimal heights [mm] & $\begin{array}{l}\mathrm{h}_{1 \min }=0,045 \\
\mathrm{~h}_{2 \min }=0,024\end{array}$ & $\begin{array}{l}\mathrm{h}_{1 \min }=0,048 \\
\mathrm{~h}_{2 \min }=0,034\end{array}$ & $\begin{array}{l}\mathrm{h}_{1 \min }=0,046 \\
\mathrm{~h}_{2 \min }=0,023\end{array}$ & $\begin{array}{l}\mathrm{h}_{1 \min }=0,047 \\
\mathrm{~h}_{2 \min }=0,036\end{array}$ \\
\hline $\begin{array}{l}\text { Velocity quotient of a bearing journal \& a } \\
\text { floating ring }\end{array}$ & $v=5,151$ & $v=3,241$ & $v=5,541$ & $v=2,311$ \\
\hline \multicolumn{5}{|l|}{ Resultant parameters, compressor side $\mathrm{F}_{\mathrm{sp}}=600[\mathrm{~N}]$} \\
\hline Structural tasks & $\begin{array}{l}1: \psi_{1}=4 \% \text { o, } \\
C_{R}^{*}=0,5\end{array}$ & $\begin{array}{l}2: \psi_{1}=4 \% \\
C_{R}^{*}=1,0\end{array}$ & $\begin{array}{l}3: \psi_{1}=5 \% \\
C_{R}^{*}=0,5\end{array}$ & $\begin{array}{l}4: \psi_{1}=5 \% \\
\mathrm{C}_{\mathrm{R}}^{*}=1,0\end{array}$ \\
\hline $\begin{array}{l}\text { Amplitudes of displacements of a floating ring } \\
\text { bearing } 10^{-6}[\mathrm{~m}]\end{array}$ & $\begin{array}{l}x_{6}=0,055 \\
y_{6}=0,190\end{array}$ & $\begin{array}{l}x_{6}=0,230 \\
y_{6}=0,900\end{array}$ & $\begin{array}{l}x_{6}=0,140 \\
y_{6}=0,353\end{array}$ & $\begin{array}{l}x_{6}=0,360 \\
y_{6}=1,160\end{array}$ \\
\hline Relative eccentricities & $\begin{array}{l}\varepsilon_{1}=0,25 \\
\varepsilon_{2}=0,19\end{array}$ & $\begin{array}{l}\varepsilon_{1}=0,22 \\
\varepsilon_{2}=0,34\end{array}$ & $\begin{array}{l}\varepsilon_{1}=0,36 \\
\varepsilon_{2}=0,36\end{array}$ & $\begin{array}{l}\varepsilon_{1}=0,31 \\
\varepsilon_{2}=0,41\end{array}$ \\
\hline Oil films maximum temperatures $\left[{ }^{0} \mathrm{C}\right]$ & $\begin{array}{c}T_{1 \max }=56 \\
T_{2 \max }=68\end{array}$ & $\begin{array}{l}T_{1 \max }=52 \\
T_{2 \max }=58\end{array}$ & $\begin{array}{l}T_{1 \max }=51 \\
T_{2 \max }=63\end{array}$ & $\begin{array}{l}T_{1 \max }=49 \\
T_{2 \max }=55\end{array}$ \\
\hline Oil films maximum pressures [MPa] & $\begin{array}{l}\mathrm{p}_{1 \max }=3,2 \\
\mathrm{p}_{2 \max }=2,2\end{array}$ & $\begin{array}{l}\mathrm{p}_{1 \max }=2,8 \\
\mathrm{p}_{2 \max }=1,8\end{array}$ & $\begin{array}{l}\mathrm{p}_{1 \max }=3,4 \\
\mathrm{p}_{2 \max }=3,6\end{array}$ & $\begin{array}{l}p_{1 \max }=3,1 \\
p_{2 \max }=1,6\end{array}$ \\
\hline Oil films minimal heights [mm] & $\begin{array}{l}\mathrm{h}_{1 \min }=0,048 \\
\mathrm{~h}_{2 \min }=0,026\end{array}$ & $\begin{array}{l}\mathrm{h}_{1 \min }=0,052 \\
\mathrm{~h}_{2 \min }=0,042\end{array}$ & $\begin{array}{l}\mathrm{h}_{1 \min }=0,051 \\
\mathrm{~h}_{2 \min }=0,025\end{array}$ & $\begin{array}{l}\mathrm{h}_{1 \min }=0,054 \\
\mathrm{~h}_{2 \min }=0,046\end{array}$ \\
\hline $\begin{array}{l}\text { Velocity quotient of a bearing journal \& a } \\
\text { floating ring bearing }\end{array}$ & $v=4,085$ & $v=2,211$ & $v=3,673$ & $v=2,171$ \\
\hline
\end{tabular}

Research article

CIndian Society for Education and Environment (iSee)
"Turbocharger: design improvement" http://www.indjst.org
A. Mazurkow Indian J.Sci.Technol. 
The analysis of the influence of structural parameters on static and dynamic properties of sliding bearings with floating rings bearing

The influence of structural parameters on static and dynamic characteristics is described in the Table 2. This kind of a description was for the first time presented for a sliding bearing with a cylindrical bush in a research work (Swiderski, 1995). The influence character of particular parameters on static and dynamic properties in slide bearings with a floating ring bearing was presented in research works (Mazurkow, 1993; Mazurkow, 2009a; Mazurkow, 2009b; Mazurkow, 2009c).

Table 2 shows that the influence of structural parameters on static and dynamic characteristics can be on one hand, contradictory e.g. the feed oil temperature and the minimal height of oil film or on the other hand, compatible e.g. the feed oil temperature and the oil film maximum temperature.

\section{Calculation examples}

To describe the influence of values of recommended parameters on work parameters of a bearing and a rotating set in a turbocharger we have accepted:

- Relative clearance of the inner oil film, $\psi_{1}=4 \%$, $\psi_{1}=5 \%$, - Quotient of radial clearances, $C_{R}^{*}=0,5, C_{R}^{*}=1,0$. Other given quantities are presented in Tables $3 \& 4$. The calculations results are described in Table 5.

\section{Conclusions}

Taking into account the results from the study we can see the essential influence of geometric parameters of sliding bearings on work parameters of a rotating set in a turbocharger. Having examined the results it can be noticed:

- Lateral sliding bearings with a floating ring will work with the following values of a relative eccentricity : $\varepsilon_{1}=<0,22 ; 0,42>, \varepsilon_{2}=<0,19 ; 054>$.

- In all examined tasks maximum temperatures and maximum pressures will have values below acceptable values. For alloys e.g. on a $\mathrm{Pb}$-base the range of their usage according to DIN 31652 amount from $\mathrm{p}_{\text {lim }}=16$ $\operatorname{MPa}\left(\mathrm{T}_{\text {lim }}=100^{\circ} \mathrm{C}\right)$ to $25 \mathrm{MPa}\left(\mathrm{T}_{\text {lim }}=50^{\circ} \mathrm{C}\right)$.

- Minimal height of lubricant films will assure a fluid friction, $h_{1,2 \mathrm{~min}}=<0,023 ; 0,054>\mathrm{mm}$.

The smallest values of displacements amplitudes of floating rings bearing appeared in the task No.1, where a relative clearance of the inner bearing and the quotient of radial clearances respectively came to: $\psi_{1}=4 \%$ and $C_{R}^{*}=0,5$. However, the biggest amplitudes of displacements were in the task No.4, for them given above values came to: $\psi_{1}=5 \%$ and $C_{R}^{*}=1,0$. The maximum, the six fold increase of amplitude of oscillations displacements appeared for a coordinate $\mathrm{y}_{6}$. In the conclusion, the authors state that geometries of sliding bearings with floating rings have a significant influence on dynamic properties of rotating sets in 